\title{
The CLS 2+1 flavor simulations
}

Piotr Korcyl*

DESY 14-224

John von Neumann Institute for Computing (NIC), DESY, Platanenallee 6, 15738 Zeuthen, Germany

E-mail: piotr.korcyl@desy.de

We report on the status of large volume simulations with $2+1$ dynamical fermions which are being performed by the CLS initiative. The algorithmic details include: open boundary conditions, twisted mass reweighting and RHMC, whereas the main feature of the simulation strategy is the approach to the physical point along a trajectory of constant trace of the mass matrix. We comment on the practical side of the above issues using as examples some of the newly generated ensembles, which presently cover lattice spacings between $0.05 \mathrm{fm}$ and $0.11 \mathrm{fm}$ and pion masses between $150 \mathrm{MeV}$ and $415 \mathrm{MeV}$.

The 32nd International Symposium on Lattice Field Theory 23-28 June, 2014

Columbia University New York, NY

${ }^{*}$ Speaker. 
In order to suppress or eliminate systematic errors associated with the continuum and chiral extrapolations, modern lattice QCD simulations try to explore the parameter space of $a \sim 0.05 \mathrm{fm}$ and physical pion mass. This is possible not only because of the increase in the available computer resources but mainly because of the progress in our understanding of numerical algorithms. New algorithmic developments are aimed at alleviating the overall scaling with $a$ and $m_{\pi}$ and try to deal with three phenomena which mainly contribute to the total cost of a simulation. These are: the autocorrelations which grow as the continuum limit is approached, partially because of the topology freezing; the accidental zero-modes of the Dirac operator which render the simulation unstable and finally, the growth of the condition number of the Dirac operator as the light quark masses are lowered. The algorithmic improvements trying to circumvent the above issues are the following:

- open boundary conditions in time [2] and periodic boundary conditions in the space directions, are supposed to let the topological charge flow in and out of the simulated volume and thus decrease the autocorrelation times,

- twisted mass infrared regulator [3] which, by moving the spectrum of the Dirac operator off the real axis, decreases the effects of the accidental zero-modes,

- deflated solver [4] which, makes the cost of the inversion largely independent of the quark mass.

The three mentioned ingredients were implemented in the open-source package openQCD [5] and used in large volume simulations with $2+1$ flavors of non-perturbatively improved Wilson fermions [1]. The simulations were made by a joint effort of the CLS [6] collaboration with computer time that was granted through two PRACE projects (at LRZ's SuperMuc in Munich (Germany) and CINECA's Fermi in Bologna (Italy)) and three national projects (Gauss-Center in Jülich (Germany), NIC in Jülich (Germany), CSCS in Lugano (Switzerland)).

In the following we present some of the first impressions gathered during these simulations. We start by briefly describing the algorithmic details, then we discuss the strategy to reach the physical point, and finally we concentrate on several observables such as $t_{0}$, the topological charge evaluated at positive Wilson flow time or the twisted mass and rational approximation reweighting factors evaluated on several of our new ensembles (for more details see Re.[1]).

\section{General setup: openQCD-1.2}

The described simulations are being made using the open-source package openQCD version 1.2 [5]. The package implements the Hybrid Monte Carlo algorithm along with several solvers enabling simulations of dynamical fermions. The gauge action used is the tree-level Symanzik improved action $\left(c_{0}=\frac{5}{3}, c_{1}=-\frac{1}{12}\right)$, while the fermion action is discretized with the Wilson $\mathscr{O}(a)$ improved action with $c_{\mathrm{SW}}$ determined non-perturbatively [7]. Open boundary conditions for the gauge fields are implemented following $[8,2]$

$$
\left.F_{0 k}(x)\right|_{x_{0}=0}=\left.F_{0 k}(x)\right|_{x_{0}=T}=0, \quad k=1,2,3,
$$


for the gauge fields and

$$
\begin{aligned}
& \left.P_{+} \psi(x)\right|_{x_{0}=0}=\left.P_{-} \psi(x)\right|_{x_{0}=T}=0, \quad P_{ \pm}=\frac{1}{2}\left(1 \pm \gamma_{0}\right) \\
& \left.\bar{\psi}(x) P_{-}\right|_{x_{0}=0}=\left.\bar{\psi}(x) P_{+}\right|_{x_{0}=T}=0,
\end{aligned}
$$

for the fermion fields. The twisted mass infrared regulator is introduced for the two degenerate light fermions [3] by replacing the original determinant $\operatorname{det}\left(D^{\dagger} D\right)$ by $\frac{\operatorname{det}\left(D^{\dagger} D+\mu_{0}^{2}\right)^{2}}{\operatorname{det}\left(D^{\dagger} D+2 \mu_{0}^{2}\right)}$ in the simulation algorithm and reweighted to the proper distribution at the analysis stage. Furthermore, the later determinant is split into typically $n \sim 5-6$ parts and each part is associated to an independent pseudofermion field,

$$
\frac{\operatorname{det}\left(D^{\dagger} D+\mu_{0}^{2}\right)^{2}}{\operatorname{det}\left(D^{\dagger} D+2 \mu_{0}^{2}\right)}=\operatorname{det}\left(D^{\dagger} D+\mu_{n}^{2}\right) \frac{\operatorname{det}\left(D^{\dagger} D+\mu_{0}^{2}\right)}{\operatorname{det}\left(D^{\dagger} D+2 \mu_{0}^{2}\right)} \prod_{k=0}^{n-1} \frac{\operatorname{det}\left(D^{\dagger} D+\mu_{k}^{2}\right)}{\operatorname{det}\left(D^{\dagger} D+\mu_{k+1}^{2}\right)}
$$

The dynamical strange quark is implemented with the RHMC algorithm with reweighting [9]. All contributions to the HMC forces are integrated along the trajectory with a set of nested MD integrators featuring a 3 level hierarchy ( $\sim 10$ steps on the coarsest level, one step on finer levels) where $2^{\text {nd }}$ (level 2) and $4^{\text {th }}$ (levels 0 and 1$)$ order Omelyan integrators are used.

\section{Strategy: our way to the physical point}

As in all such projects, one has to choose the starting point in parameter space and define the trajectory along which the continuum and chiral limits are taken. To do so, we define two dimensionless quantities which are used to perform the matching:

$$
\begin{aligned}
& \phi_{2}\left(\beta, \kappa_{u}, \kappa_{s}\right)=8 t_{0} m_{\pi}^{2} \propto m_{u d}+\mathscr{O}\left(m_{u d}^{2}\right) \\
& \phi_{4}\left(\beta, \kappa_{u}, \kappa_{s}\right)=8 t_{0}\left(m_{K}^{2}+\frac{1}{2} m_{\pi}^{2}\right) \propto \operatorname{tr} M+\mathscr{O}\left(\operatorname{tr} M^{2}\right)
\end{aligned}
$$

Our trajectory is defined by keeping the trace of the mass matrix constant, namely

$$
\sum_{i=u, d, s} \frac{1}{\kappa_{i}}=\text { const } \Leftrightarrow \operatorname{tr} M_{\mathrm{R}}=\text { const }+\mathscr{O}(a)
$$

In particular, at the symmetric point $\kappa_{u}=\kappa_{d}=\kappa_{s}$ one is left with only one free parameter and therefore the matching for different lattice spacings can be done relatively easily. Figure 1 presents the results of the performed matching. The three points on the right (with $\phi_{2} \approx 0.75$ ) come from the three matched ensembles at the symmetric point, whereas the remaining data points have different values of $\phi_{2}$ but should lie along the line of $\phi_{4} \approx$ const. The estimate of the physical point uses the values of $m_{\pi}$ and $m_{K}$ taken from PDG and $\sqrt{t_{0}}=0.1465 \mathrm{fm}$ from [10].

\section{First impressions}

During the granted computer time 10 ensembles were generated with MC chains of length of at least $50 \tau^{e x p}$, whose details are summarized in table 1 (for more information see Ref.[1]). Simultaneously with the generation of configurations several quantities were monitored, such as the topological charge and the action density at positive Wilson flow time and the two reweighting factors. We now discuss them in more detail. 


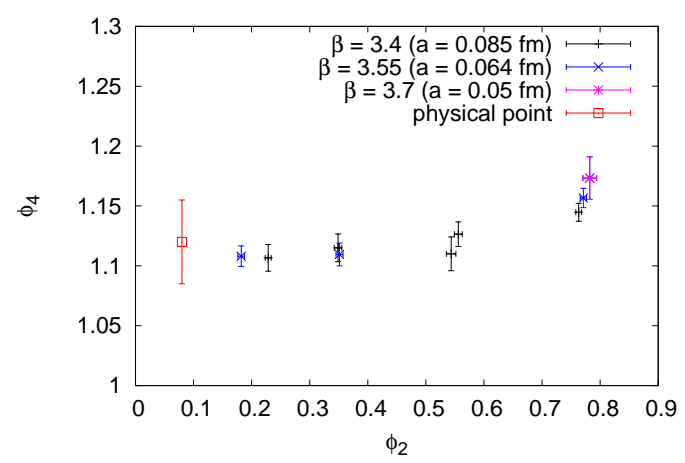

Figure 1: Tuning to the trajectory of constant trace of the mass matrix.

\begin{tabular}{|cc|cccc|}
\hline & & $0.085 \mathrm{fm}$ & $0.064 \mathrm{fm}$ & $0.05 \mathrm{fm}$ & $\mathrm{a}[\mathrm{fm}]$ \\
$m_{K}$ & $m_{\pi}$ & 3.4 & 3.55 & 3.7 & $\beta$ \\
\hline $415 \mathrm{MeV}$ & $415 \mathrm{MeV}$ & $32^{3} \times 96$ & $32^{3} \times 96$ & $48^{3} \times 128$ & \\
$440 \mathrm{MeV}$ & $350 \mathrm{MeV}$ & $32^{3} \times 96$ & & & \\
$470 \mathrm{MeV}$ & $280 \mathrm{MeV}$ & $32^{3} \times 96$ & $48^{3} \times 128$ & $64^{3} \times 192$ & \\
$480 \mathrm{MeV}$ & $220 \mathrm{MeV}$ & $48^{3} \times 96$ & $64^{3} \times 128$ & & \\
$490 \mathrm{MeV}$ & $150 \mathrm{MeV}$ & $64^{3} \times 128$ & & & \\
\hline
\end{tabular}

Table 1: Overview table of lattice spacings and pion masses. The red entries correspond to the ensembles at the symmetric point that were used in the matching between different lattice spacings. The blue entries correspond to the ensembles being still generated.

\subsection{Reweighting factors}

Before an average value of any physical observable is computed, one has to measure the twisted mass and rational reweigthing factors, used to be denoted by $W_{0}$ and $W_{1}$, respectively. They are given by

$$
W_{0}=\frac{\operatorname{det}\left(D^{\dagger} D\right) \operatorname{det}\left(D^{\dagger} D+2 \mu^{2}\right)}{\operatorname{det}\left(D^{\dagger} D+\mu^{2}\right)^{2}}, \quad W_{1}=\operatorname{det}\left(D_{s} R\right)
$$

with $Z=D_{s}^{\dagger} D_{s} R^{2}-1$, where $\operatorname{det} D_{s}=W_{1} \operatorname{det} R^{-1}$ and $R$ is the Zolotarev rational approximation. We stress that the inaccuracy of the rational approximation is dealt with in these simulations by reweighting instead of an additional Metropolis step as is done typically.

The left and middle panels of figure 2 show samples of MC histories of the twisted mass reweigthing factor for different ensembles. Their values fluctuate close to 1 as expected. From the algorithmic point of view there is nothing wrong with having $W_{0}$ close to or equal to zero, however such small reweighting factors may lead to a decrease of the precision of the final average [12], therefore they have to be monitored during the entire production phase.

As far as the rational reweighting factors are concerned, the right panel of figure 2 shows extracts of MC histories of $W_{1}$ for the three ensembles at the symmetric point at three different 

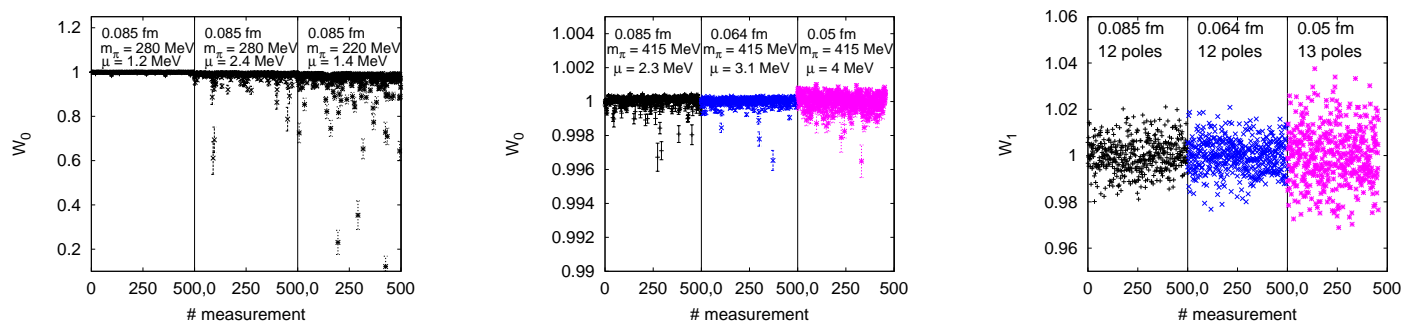

Figure 2: Histories of the reweigthing factors. On the left plot we compare $W_{0}$ of the three ensembles having the same lattice spacing but different $m_{\pi}$ or $\mu$, the plot in the middle shows the comparison of $W_{0}$ evaluated at the symmetric ensembles at different lattice spacings, whereas the plot on the right shows the histories of the rational approximation reweighting factor at the symmetric point. Plotted data comes from ensembles: left panel: H105r005, H105r001, C101r014; middle panel: H101r001, H200r000, N300r002; right panel: H101r001, H200r000, N300r002, see Ref.[1].
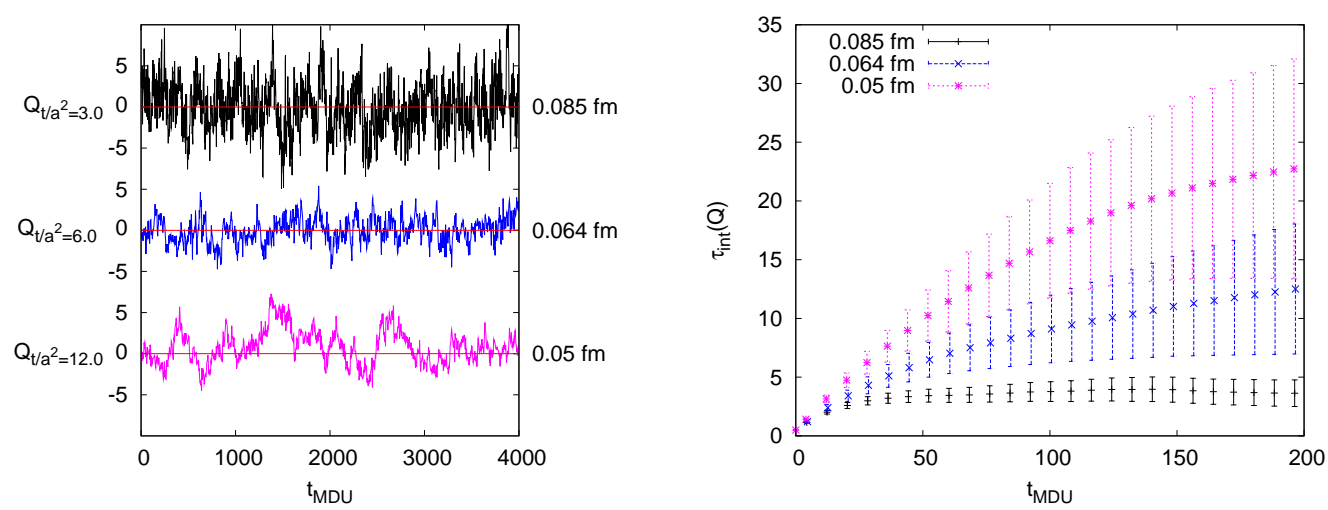

Figure 3: Topological charge at the symmetric point at flow times $t / a^{2}=3.0,6.0,12.0$ which roughly reflect the scaling of $t_{0}$ with $a$. Data comes from ensembles H101r001, H200r000 and N300r002, see Ref.[1].

lattice spacings. We see that the fluctuations are very small and do not depend significantly on the lattice spacing. One concludes that the reweighting of the rational approximation works very well.

\subsection{Physical observables}

Once the reweighting factors are computed one can evaluate expectation values of physical observables. First, we compute the topological charge given by the field-theoretic definition at Wilson flow time of the order of $t_{0}$. The left panel of figure 3 shows the comparison of the history of the topological charge at three different lattice spacings evaluated on ensembles at the symmetric point. Similar information is plotted on the right panel of that figure, where the integrated autocorrelation times are shown. One clearly sees an increase of $\tau_{\text {int }}$ as $a$ is decreased with a scaling in agreement with [2].

Next, we discuss the YM action density evaluated at Wilson flow time of the order of $t_{0}$. Figure 

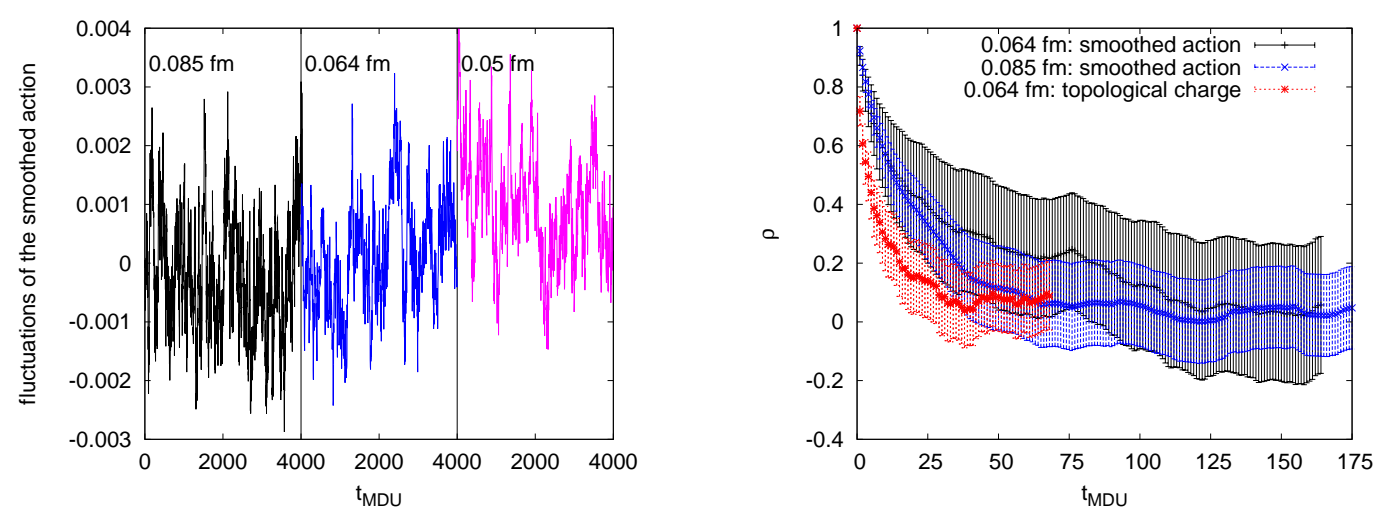

Figure 4: The left panel shows the fluctuations of the YM action density at the symmetric point at flow time $t / a^{2}=3.0,6.0,12.0$. The right panel shows the comparison of autocorrelation functions of $E(t)$ and $Q(t)$. Data comes from ensembles H101r000, H200r000 and N300r002 (only part of the full statistics is shown), see Ref.[1].

4 compares the MC history of the fluctuations of this observable for three different lattice spacings. Figure 4 also shows the autocorrelation functions of $E(t)$ corresponding to the two coarsest ensembles together with the autocorrelation function of the topological charge. We notice that the integrated autocorrelation time of the topological charge is smaller, which is consistent with the recent findings of [11].

$E(t)$ evaluated at each time-slice independently can be use to exhibit cut-off effects introduced by the open boundaries. On figure 5 we plot $E(t)$ for different ensembles as a function of physical distance from the boundary expressed in terms of $x_{0} / \sqrt{t_{0}}$. The value approaches 0.3 by difinition in the central region of the lattice. We see that the height of this lattice artifact depends strongly on the lattice spacings (the three distinctive sets of data points) and is roughly independent of the pion mass (different colors of the data points within each set). We also included results from a simulation with a Iwasaki gauge action at a lattice spacing of $a \approx 0.09 \mathrm{fm}$. The discrepancy between these data and the data obtained with the Wilson action with a similar lattice spacing confirms the cut-off nature of the visible structure. On the right of figure 5 we show the dependence of $E\left(t, x_{0}\right)$ on $a^{2}$ for three different values of $x_{0} / \sqrt{t_{0}}$. The data cannot be described by a linear dependence on the lattice spacing.

Finally, we present our estimates of $t_{0}$ for all the considered ensembles normalized by the value obtained at the symmetric point as a function of $m_{\pi}$, see figure 6 . We also include in the plot the analytic prediction from NLO chiral perturbation theory worked out in [13]. We notice that the data points deviate from this prediction by very small amounts; cut-off effects are small.

\section{Conclusions}

Several points should be highlighted. The algorithmic improvements turned out to work very well in practice. In all our runs the algorithm proved to be stable. The first preliminary measurements of physical observables show that the expected precision can be reached. The trajectory of 

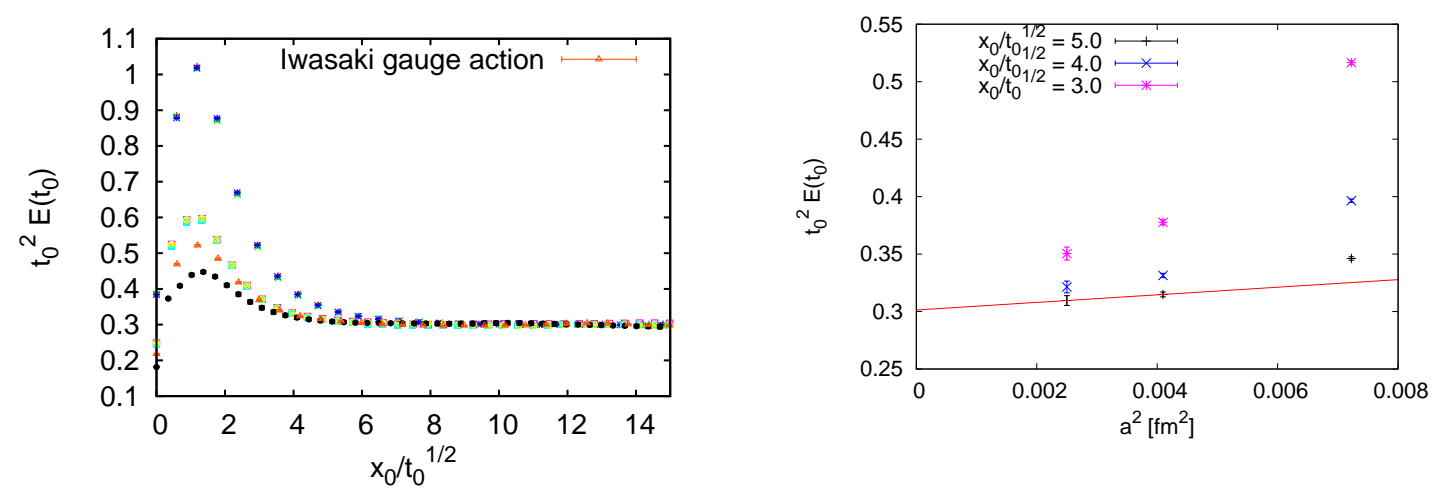

Figure 5: $E\left(t_{0}, x_{0}\right)$ as a function of the distance from the boundary shows large cut-off effects. They exhibit strong lattice spacing dependence (see plot on the right), but are largely independent of the pion mass.

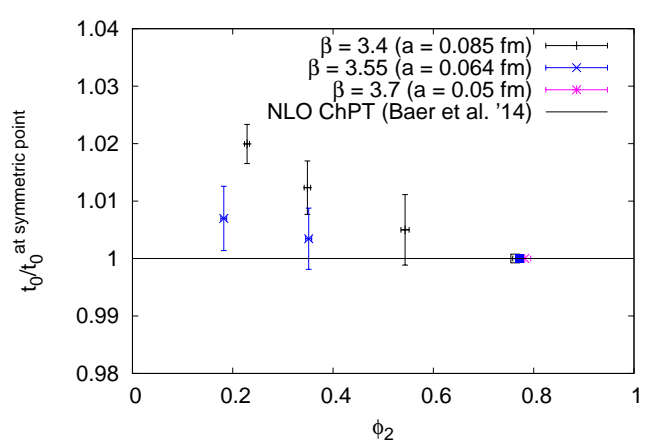

Figure 6: Dependence of $t_{0} / t_{0}^{\text {ref }}$ on $m_{\pi}$ at different lattice spacings. The data show that the cut-off effects in this observable as well as its pion mass dependence are small, which is favorable in view of the tunning to the chiral trajectory.

constant trace of the mass matrix allowed precise matching and the tunning to the chiral trajectory turned out to be possible with small effort. The autocorrelation time of the topological charge is not the largest one, in agreement with Ref.[11].

\section{Acknowledgments}

The author would like to thank all the CLS collaboration and in particular M. Bruno, T. Korzec and S. Lottini for the help with the figures and S. Schaefer, R. Sommer and H. Simma for many useful discussions.

\section{References}

[1] M. Bruno et al., arXiv:1411.3982 
[2] M. Lüscher, S. Schaefer, JHEP 1107 (2011) 036, arXiv:1105.4749

[3] M. Lüscher, F. Palombi, PoS LATTICE2008 (2008) 049, arXiv:0810.0946

[4] M. Luscher, Comput. Phys. Commun. 156 (2004) 209-220, arXiv:hep-lat/0310048v1

[5] http://luscher.web.cern.ch/luscher/openQCD/

[6] Coordinated Lattice Simulations, https://wiki-zeuthen.desy.de/CLS/CLS

[7] J. Bulava, S. Schaefer, Nucl.Phys. B874 (2013) 188-197, arXiv:1304.7093

[8] M. Lüscher, PoS LATTICE2010 (2010) 015, arXiv:1009.5877

[9] M. Lüscher, S. Schaefer, Comput.Phys.Commun. 184 (2013) 519-528, arXiv:1206.2809

[10] S. Borsanyi et al., JHEP 1209 (2012) 010, arXiv:1203.4469

[11] M. Bruno et al., JHEP 1408 (2014) 150, arXiv:1406.5363

[12] M. Bruno et al., arXiv:1411.5207, in these proceedings

[13] M. Golterman, O. Bär, Phys.Rev. D89 (2014) 034505, arXiv:1312.4999 